\title{
Closed Loop Subspace Identification and Control of a two input-Single output Conical Tank System
}

\author{
Sudhahar S 1,*, Ganesh Babu $C^{2}$ and Sharmila $D^{3}$ \\ 1,* Bannari Amman Institute of Technology; sudhahars@bitsathy.ac.in \\ 2 Bannari Amman Institute of Technology; ganeshbabuc@ bitsathy.ac.in \\ 3 Jai Shriram Engineering College; sharmiramesh@rediffmail.com \\ * Correspondence: sudhahars@bitsathy.ac.in;
}

\begin{abstract}
The process model is very essential for the model based control design. The model of the process can be identified using system identification algorithm. The system identification is done through the open loop and closed loop approaches. In this work, the lab scale conical tank setup configured as a non square MIMO system. The conical tank system is identified through the both appraoches, the effectiveness and need of the both approaces are discussed. Based on the open loop identified model the controller designed and the controller implemented in the real time the to record the process data. From this data the closed loop identification are conducted uisng N4SID algorithm. The controller seetings are obtained using the smith predcitor based IMC based PI controller for the obtained model. The proposed identification algorithm and controller tuning show the better reults over the conventional method. Moreover, this method is applicable for all the non square MIMO system.
\end{abstract}

Keywords: System identification; PI control; System Modeling; MISO; Performance analysis.

\section{Introduction}

The measure of correlation between the past and conditioned future inputs is computed using the canonical variate analysis (CVA) [10]. The linear time invariant MIMO process can be identified using Multivariable Output-Error State Space (MOESP) in the presence of process noise and measurement noise and the algorithm is based on the class of instrumental variables [17]. The sub space based system identification method is outperformed then the prediction error method for the appropriate input design [18]. The closed loop system identification method having the following advantages over the open loop system identification method, (i) The plant model can be obtained with greater accuracy for controller design; (ii) The ease of controller re-tuning, (iii) Controller model order reduction is possible without affecting the inherent properties of the closed loop system, (iv) Controller maintenance and Validation [9]. Some of the process control applications are prohibited for open loop system identification, due to the following reasons; (i) The system is isolated from the field for a particular time interval so that the stability of the system may get affected, (ii) The isolation may cause the safety problems in the field, (iii) The isolation of the system may pay high operational cost, (iv) The system may unstable under open loop conditions, so the feedback mechanism is required to stabilize the system. The main drawback of the closed loop system identification method is the process noise and input are correlated $[5,19]$. The errors-in-variable based Maximum Likelihood method and the Least Square Estimation method are easy and efficient in open loop system identification. The methods are applied to the closed loop identification directly then the estimation gives a large bias [2]. The Laguerre model based closed loop system identification algorithm is performed for the step change input [20]. The closed loop system identification methods are classified based on the number of stages and each method having their own merits [11]. The plant and controller is identified by identifying the deterministic part by using a subspace method of closed loop identification with the joint input-output framework [6]. The hybrid parameter estimation is calculated by using Monte Carlo simulation analysis. Here, the 
measurement input and output are considered as a continuous time transfer function and noise is represented in the form of discrete time auto regressive process [21]. Economical input was designed for system identification of multivariate systems. The identification cost is minimized through the optimal input design without sacrificing the operational constraints and the quality of model [1]. The cost of control effort is minimized through the convex optimization on-line on the implemented state feedback law which uses an all the measurements inputs and outputs to linearize the system [7]. The Internal Model Control (IMC) used to design controller for non square MIMO system using Smith delay compensation method [3]. The air path of a turbocharged diesel engine is identified through the frequency domain system identification and control using CRONE controller which ensure the robust performance and stability [8]. The direct internal model controller combined with Smith delay compensator designed for non-square MIMO system with delay times [13]. Exponentially Weighted Moving Average (EWMA) controller is designed for non square MIMO process [14]. The Non-Dimensional Tuning (NDT) method gives the better results than the SIMC method and pole placement method in terms of settling time, minimum overshoot, and less interaction [15]. The closed loop system identification methods are classified based on their characterization: direct, indirect and joint input-output approach [4]. (i) The direct approach: The system identified by using process input and output of the process, here the feedback and reference signal are not considered. (ii) The Indirect approach: In this method the system is identified using reference signal and process output, and plant model is recovered using known controller settings. (iii) The Joint Input-Output Approach: The process output and input combined as output data for the identification method and reference signal and noise signal are considered as an input to the system. Knowledge of the controller settings the system model is obtained. In this paper the closed loop system identification method only considers the process inputs and outputs only, and the controller and feedback are ignored. Based on the open loop model the PI control is derived. With the known controller settings, the inputs from the controller to the process considered as an instrumental variable and this variable is not correlated with noises for closed loop state space subspace system identification.

\section{System Description}

The state space model of the multi input single output system is represented by the following equations.

$$
\begin{aligned}
& x_{k+1}=A x_{k}+B u_{k}+\omega_{k} \\
& y_{k}=C x_{k}+D u_{k}+v_{k}
\end{aligned}
$$

Where $\mathrm{A}, \mathrm{B}, \mathrm{C}$, and D are system, input, output and transmission matrices with appropriate dimensions. $x_{k} \in \mathfrak{R}^{n}, u_{k} \in \mathfrak{R}^{l}$ and $y_{k} \in \mathfrak{R}^{m}$ respectively represent the vectors of the system state,

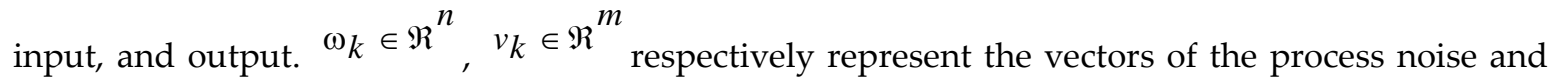
measurement noise.

The innovation model of the state space model is a steady Kalman filter:

$$
\begin{aligned}
& \hat{x}_{k+1}=A \hat{x}_{k}+B u_{k}+K e_{k} \\
& y_{k}=C \hat{x}_{k}+D u_{k}+e_{k}
\end{aligned}
$$

Where $\hat{x}_{k}$ the model of the state vector, $\mathrm{K}$ is the gain matrix of the Kalman filter, ek is a white residual sequence and the assumption made that the sequence is uncorrelated with past input and output measurements.

The equivalent predictor model is formulated using (3) and (4):

$$
\begin{aligned}
& \hat{x}_{k+1}=A_{K} \hat{x}_{k}+B{ }_{K} u_{k}+K y_{k} \\
& y_{k}=C \hat{x}_{k}+D u_{k}+e_{k} \\
& \text { Where }{ }^{A}=A-K C, B_{K}=B-K D
\end{aligned}
$$


The extended state-space model of the process computed using the state space model equations (1) \& (2):

$$
\begin{aligned}
& Y_{f}=\Gamma_{f} X_{k, l}+H_{u, f} U_{f}+\Phi_{f} \\
& \text { Where } \Phi_{k}=H_{w, f} W_{f}+V_{f} \\
& \Gamma_{f} \text { is the extended observability matrix. }
\end{aligned}
$$

The extended innovation state-space model can be recognized as [12].

$$
Y_{f}=\Gamma_{f} \hat{X}_{k, l}+H_{u, f} U_{f}+H_{e, f} E_{f}
$$

The extended predictor state-space model is formulated as

$$
\left(I-H_{y, f}^{K}\right) Y_{f}=\Gamma_{f}^{K} \hat{X}_{k, l}+H_{u, f}^{K} U_{f}+E_{f}
$$

The MISO system is identified using CN4SID (closed-loop numerical algorithms for subspace state space system identification).

\section{Closed Loop System Identification Algorithm}

The steps of closed loop system identification algorithm are summarized in the table 1 . The state space model of the process model is represented in equation (1) and (2) with the standard feedback control. The controller state space model can be represented as follows:

$$
\begin{aligned}
& x_{k+1}^{c}=A_{c} x_{k}^{c}+B_{c}\left(r_{k}-y_{k}\right) \\
& u_{k}=C_{c} x_{k}^{c}+D_{c}\left(r_{k}-y_{k}\right)
\end{aligned}
$$

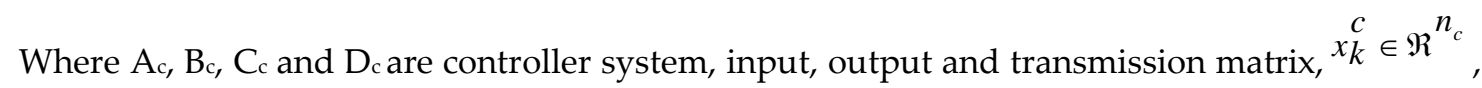
$r_{k} \in \mathfrak{R}^{m}$ represents the vectors of controller state and the reference input. The following assumptions are made to proceed with the consequent results:

1) The standard feedback controller is implemented in the control loops and the system stabilized with the controller settings.

2) The guided input signal $r_{k} \in \mathfrak{R}^{m}$ is not correlated in the closed loop with the conical tank system process noise and measurement noise $\omega_{k} \in \mathfrak{R}^{n}$ and $v_{k} \in \mathfrak{R} m$.

3) The control output / the process input $u \in \mathfrak{R}^{l}$ should satisfy the persistent excitation condition.

4) The conical tank system process noise and measurement noise $\omega_{k} \in \Re^{n}$ and $v_{k} \in \mathfrak{R}^{m}$ should be white noises subject to the normal distribution.

5) The data of guided signal $\left(\mathrm{r}_{\mathrm{k}}\right)$, controller output $(\mathrm{uk})$, and process output $(\mathrm{yk})$ are observed during the process run time and run time should be sufficiently large.

\section{Table 1}

Closed-Loop System Identification Algorithm

Step 1: Conduct the open loop test on the plant and collect the input $u_{k}$ and output data yk. Step 2: Identify the model of the system using N4SID identification algorithm.

Step 3: Compute the PI controller settings for MISO system.

Step 4: Construct the closed loop system by implementing PI controller as a cascade controller.

Step 5: Conduct the closed loop test on the plant and collect the reference $\mathrm{rk}_{\mathrm{k}}$, controller output $\mathrm{uk}$ and output of the plant $\mathrm{yk}$. 
Step 6: Determine the parameter value of $\mathrm{sp}_{\mathrm{p}} \mathrm{sf}$ and $\mathrm{N}$.

Step 7: Construct the Henkel Matrix: $U_{p}=U_{k-s_{p}, s_{p}}, U_{f}=U_{k, s_{f}}, Y_{p}=Y_{k-s_{p}, s_{p}}$, $Y_{f}=Y_{k, s_{f}}, R_{p}=R_{k-s_{p}, s_{p} \text { and }} R_{f}=R_{k, s_{f}}$

Step 8: Construct the matrix $H_{u, f}^{c}$ and $H_{u, p}^{c}$

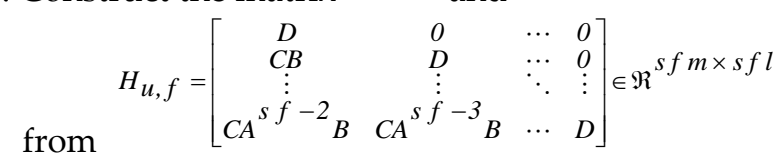

Step 9: Compute $\mathrm{M}_{\mathrm{f}}$ and $\mathrm{M}_{\mathrm{p}}$.

$$
\begin{aligned}
& M_{f}=U_{f}+H_{u, f}^{c} Y_{f} \\
& M_{p}=U_{p}+H_{u, p}^{c} Y_{p}
\end{aligned}
$$

Step 10: Conduct the LQ factorization

$$
\begin{aligned}
& {\left[\begin{array}{c}
Z_{c, p} \\
M_{f} \\
Y_{f}
\end{array}\right]=\left[\begin{array}{ccc}
L_{c, 11} & 0 & 0 \\
L_{c, 21} & L_{c, 22} & 0 \\
L_{c, 31} & L_{c, 32} & L_{c, 33}
\end{array}\right]\left[\begin{array}{l}
Q_{c, 1} \\
Q_{c, 2} \\
Q_{c, 3}
\end{array}\right]} \\
& Z_{c, p}=\left[\begin{array}{c}
M_{p} \\
Y_{p}
\end{array}\right]
\end{aligned}
$$

Step 11: Compute the $H_{u, f} \& \Gamma_{f} X_{k, 1}$

$$
\begin{aligned}
& H_{u, f}=T_{f} L_{c, 32} L_{c, 22}^{-1} \\
& \Gamma_{f} X_{k, l}=\left(I-L_{c, 32} L_{c, 22}^{-1} H_{u, f}^{c}\right)^{-1} \times\left(L_{c, 3} L_{C, 11}^{-1}-L_{C, 21} L_{C, 11}^{-1}\right) z_{c, p}
\end{aligned}
$$

Step 12: Compute the A, B, C, and D of the plant.

$$
\begin{aligned}
C & =\Gamma_{f, 1}:\left(s_{f}-1\right) m(1: m, 1: n) \\
A & =\Gamma_{f, 1:}^{+}:\left(s_{f}-1\right) m \Gamma_{f, m+1: s_{f} m} \\
D & =H_{u, f}\left(\left(s_{f}-1\right) m+1: s_{f} m,\left(s_{f-1}\right)+1: s_{f} l\right) \\
B & =\Gamma_{f, 1:\left(s_{f}-1\right) m}^{+} H_{u, f}\left(m+1: s_{f} m, 1: l\right)
\end{aligned}
$$

\section{Process Description}

For experimental study the conical tank process is considered, and shown in the Figure: 1 . The experimental setup has the three conical tanks in which process tank 1 is considered. It has a reservoir tank to accumulate the water for carry out experiments. The experimental setup configured as a MISO system by the following formulation: The level of the process tank 1 considered as a process variable (PV) and the corresponding inputs/controller outputs $1 \& 2$ considered as a manipulated variables 1 (MV1) \& 2 (MV2). So the process tank 1 level is varied by both of the inputs, hence it is formed two input one output system. The single phase motor is excited by $230 \mathrm{~V}$ AC supply from mains. The pneumatic control valves are actuated through the signal obtained from $\mathrm{I} / \mathrm{P}$ converter (3 to $15 \mathrm{psi}$ ) is used to operate the pneumatic valve and the valve regulates the flow rate based on the digital control output. The I/P converter is fed by current signal and is converted by current corresponding pressure. The pressure is obtained from the compressor and it is regulated by the air regulator. The I/P converter generates a 3 to 15 Pounds per Square Inch (psi) signal proportional to a current signal of 4 to $20 \mathrm{~mA}$. The current in the I/P converter is obtained from the $\mathrm{V} / \mathrm{I}$ converter because the output of the DAQ card is voltage, so the voltage is converted to current. 
The DAQ card is interfaced with PC through LabVIEW software. The control logic and manipulation are coded in the LabVIEW. The level of conical tanks is measured by Differential Pressure Transmitter (DPT) that is Level Transmitter (LT).

The conical pilot plant has the several field components, which are connected as shown in Figure: 1; for the measurement and control purpose. The field instruments are calibrated for the accuracy and reliability of the instrument readings and to ensure readings from an instrument are consistent with other measurements.

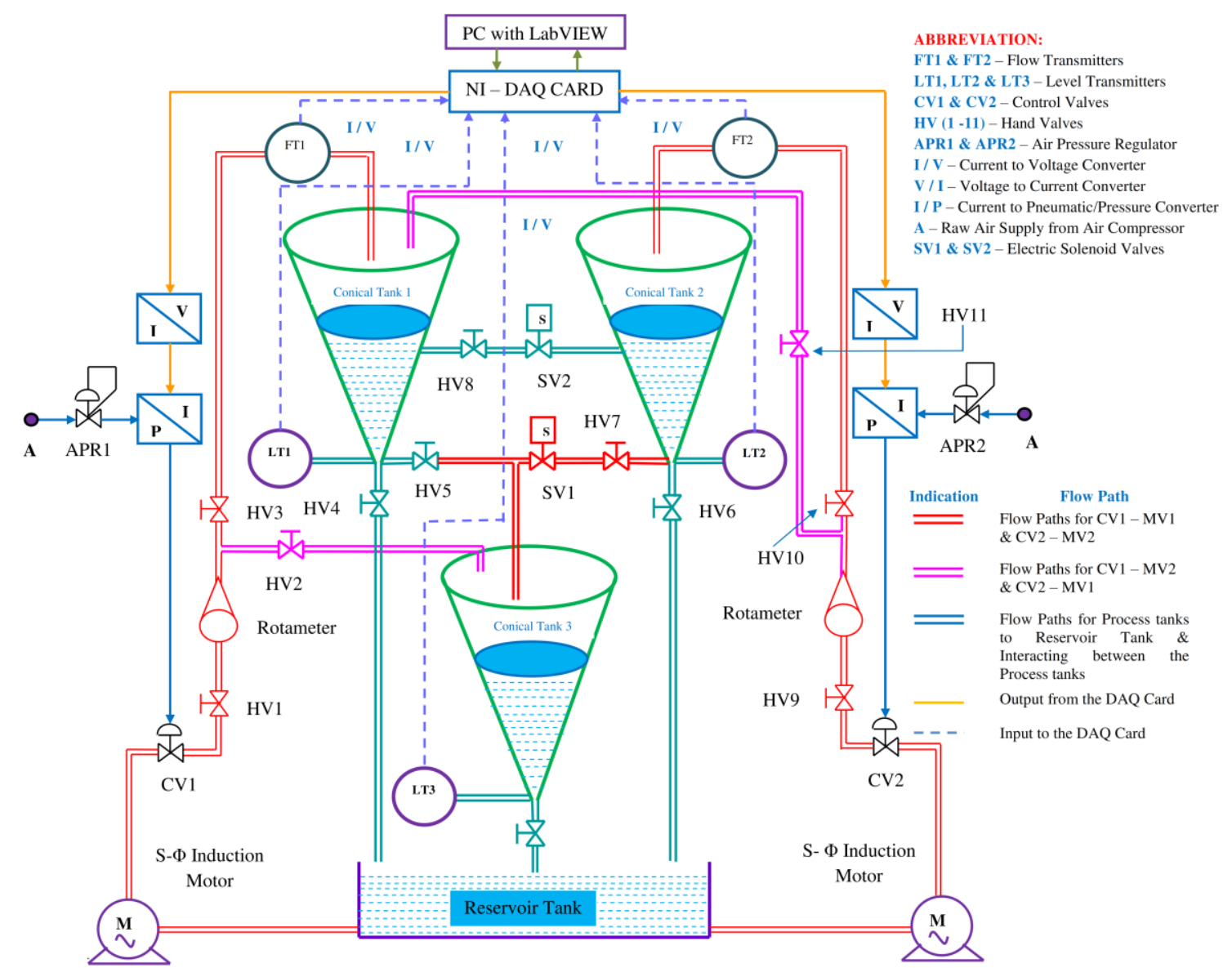

Figure: 1 . Three tank conical system

\section{Model Structure}

From the analysis of conical tank plant, response from the each control valves by the corresponding manipulated variable. However the level in the conical tank affected by the both control valves; the dynamics of plant operation in open loop is demonstrated in Figure: 2 . Where Gij represents the transfer function of ith input to jth output, ui is the input signal and yj represents the output of the system which is varied by two input variables.

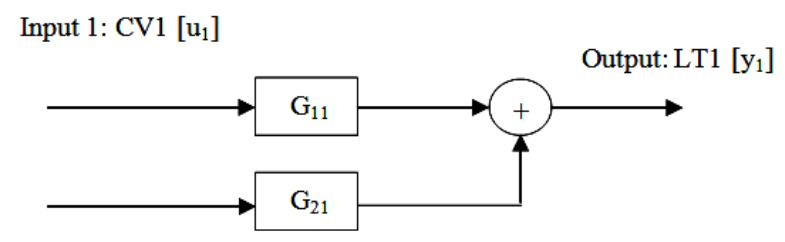

Input 2: CV2 $\left[\mathrm{u}_{2}\right]$

Figure: 2. Open Loop Structure

Figure: 2, represent the open loop structure of the plant, then the dynamics of the plant can be written as 


$$
y=\left[\begin{array}{ll}
G_{11} & G_{21}
\end{array}\right]\left[\begin{array}{l}
u_{1} \\
u_{2}
\end{array}\right]
$$

The configuration of MISO in closed loop is shown in Figure: 3. The open loop identification method is conducted on the cnicaal tank to identify the plant of the system. The PI controller designed using relay feedback method and implmented in the plant. To identify the system in closed loop the manipulated variable or reference variable taken as input. If the manipulated taken as input, then the external excitation is required as shown in Figure: 3. Where Gij represents the transfer function of ith input to jth output, ui is the input signal, Ri reference input signal, Ej is the external excitation signal, PI-I \& PI-II is the controller settings of the control valves 1 and $2, \mathrm{Dj}$ is the transfer function of the external excitation or the disturbance transfer function, and yj represents the output of the system which is varied by two input form.

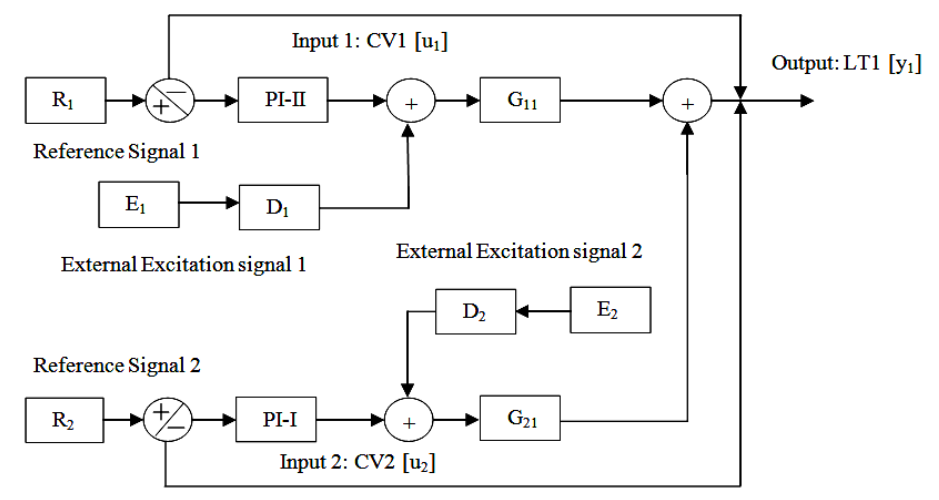

Figure: 3. Closed Loop Structure

\section{System Identification Process}

The non linear system is identified using the system identification method by designing the proper input design. The input signal should cover all the ranges of magnitudes and frequencies. The input signal should satisfy the persistent excitation conditions so that the variables of input should be parameterized. Here, the Amplitude-modulation Pseudo Random Binary Signal (APRBS) is chosen for system identification which is satisfied above mentioned criteria. The equation of APRBS signal is given by

$$
\begin{aligned}
& u=u_{O}+\sum\left(a_{i}-a_{i-1}\right) h\left(t-t_{i}\right) \\
& h\left(t-t_{i}\right)= \begin{cases}1 & t \geq t_{i} \\
0 & t<t_{i}\end{cases}
\end{aligned}
$$

So, APRBS is selected for the identification and validation purpose. The two different APRBS signal are designed, and are generated by the NI DAQ card as a voltage, subsequently converted to current by V to I converter, this current is converted to pressure by I to P converter, this pressure given to input as the two control valves concurrently as the plant inputs. The level of the conical tank is recorded with one sampling time. From the measured data the model was identified in two methods. Model 1 is developed by using N4SID subspace algorithm plant under open loop condition for controller setting. Model 2 \& Model 3 are developed by using SIMPCA and N4SID subspace algorithm plant under closed loop condition with controllers. The input signals are shown in Figure: 4 \& 5, these signals are used in both open and closed loop identification techniques. However, the system identified in two ways different input signals or same input signals. 


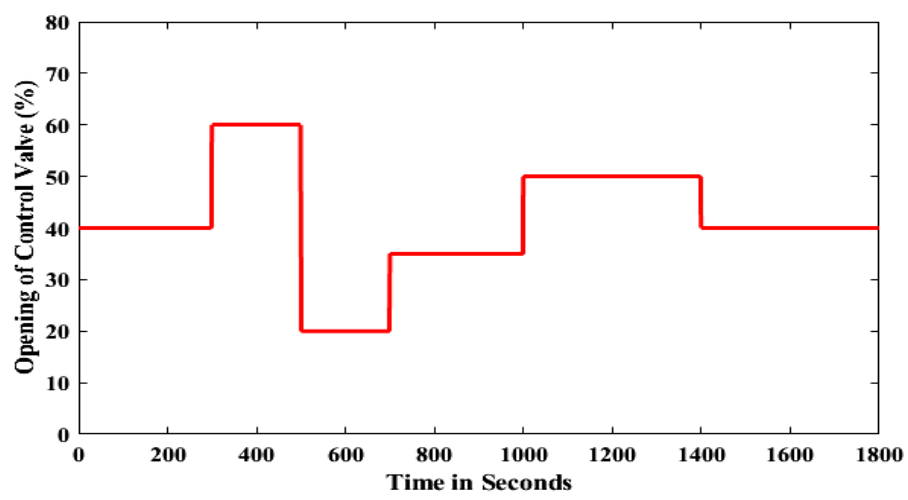

Figure: 4 . Input Signal 1

The order of the state space model was determined by the singular value decomposition in N4SID algorithm. Figure: $6 \& 7$ shows the suggests the order of the system in both loops as $1 \& 2$ respectively based on the outcomes of singular value decomposition which is carry out by N4SID algorithm.

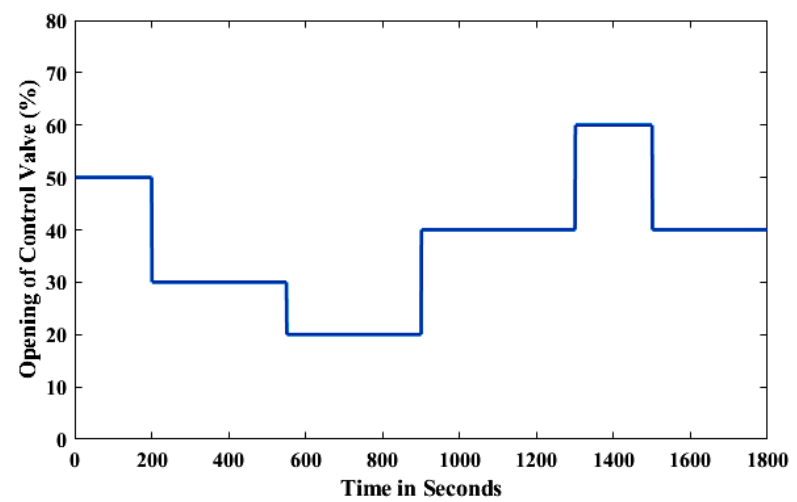

Figure: 5. Input Signal 2

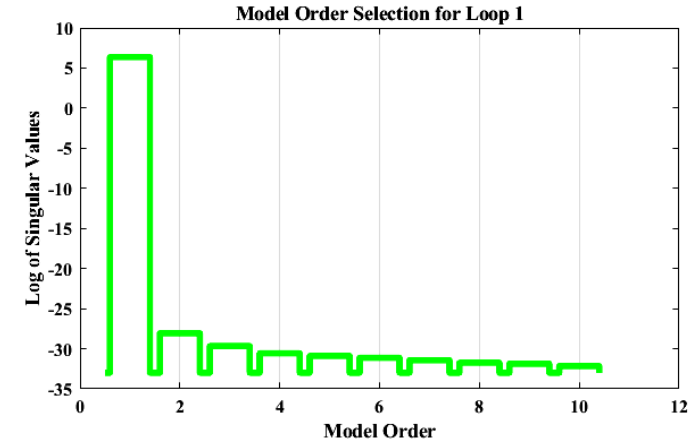

Figure: 6. Model Order Selection for Loop 1

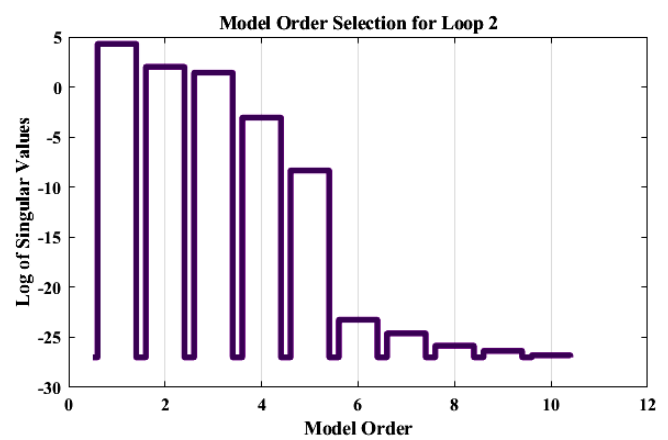

Figure: 7. Model Order Selection for Loop 2 
The simulated output which is generated by the two models and the real plant output are compared to the APRBS input signal for the level of the conical tank 1 is shown in Figure: 8 and Figure: 9 respectively.

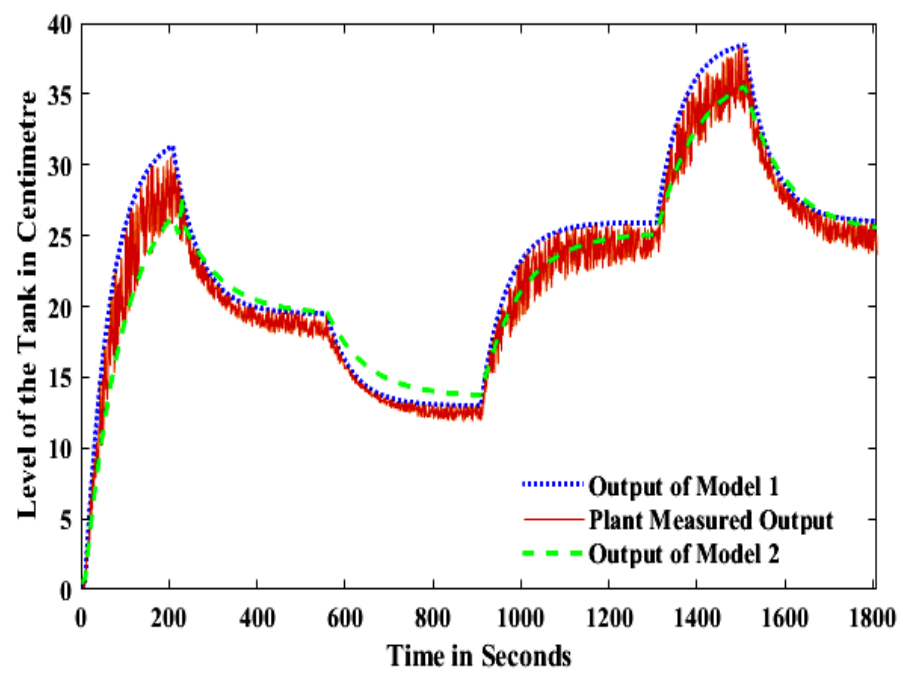

Figure: 8. Measured and Simulated output Loop 1

The real plant output and the output of the models ( $1 \& 2$ ) output to the APRBS signal for the tank level through loop 1 is shown in Figure: 8.

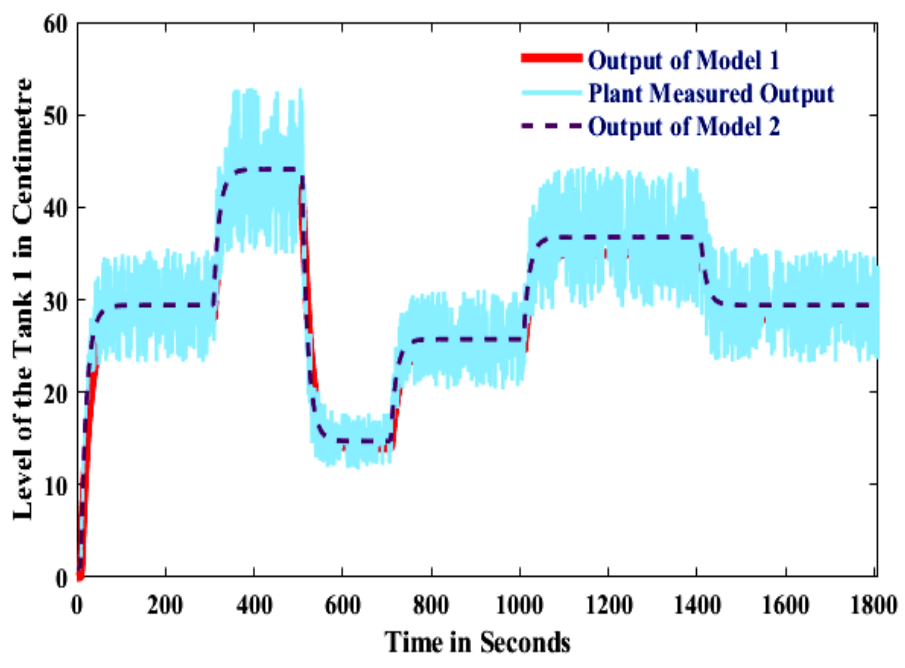

Figure: 9. Measured and Simulated output Loop 2

The real plant output and the output of the models ( $1 \& 2$ ) output to the APRBS signal for the tank level through loop 2 is shown in Figure: 9.

The design of PI controllers for the developed model and implemented in real time for closed loop system identification. Two PI controllers' settings are computed to control the level of the process tank 1 . The controller settings are given in equation (15) \& (16)

$$
\begin{aligned}
& C_{1}(s)=0.625\left\{e_{i}(t)+\frac{1}{0.0232} \int_{0}^{t} e_{i}(\tau) d \tau\right\} \\
& C_{2}(s)=7.145\left\{e_{i}(t)+\frac{1}{0.0685} \int_{0}^{t} e_{i}(\tau) d \tau\right\}
\end{aligned}
$$


The same input signals are utilized to identify the system in closed loop system identification, and the experimental setup is shown in Figure: 3, the input moves are determined by the controller; so there no direct way to alter the input signal. However, indirect method available to excite the process through set point (input) or an external signal that is introduced at the input or at the set point. The design of experiments for identification should have identifiability from the input and output data. From the experiment, the reference $r_{k}$, controller output $u_{k}$ and output of the plant $y_{k}$ data's are collected. The parameter value of $\mathrm{Sp}, \mathrm{Sf}$ and $\mathrm{N}$ are chosen as 45,10 and $1.809 \times 10^{3}$ respectively. The process data is splitted into past and future horizon; this is formed by Henkel's matrix. LQ factorization is conducted using the extended observability matrix which has two lower Toeplitz matrices, and known controller settings. Finally, the state-space model conical tank system in the process form is formulated.

\section{Validation of Model}

To investigate the effectiveness of the model the best fit criterion is used. This shows the how the plant model reproduces the with measured plant data.

$J_{B F R}=\left(1-\frac{\sqrt{\sum_{k=1}^{N}(y(k)-\hat{y}(k))^{2}}}{\sqrt{\sum_{k=1}^{N}(y(k)-\bar{y})^{2}}}\right) \times 100$

$\bar{y}$ is the mean of the measured output.

The APRBS signals shown in Figure: $4 \& 5$ are given as input to the actuators (control valves) independently. The process data are recorded with the sampling rate of one second. The simulated output which is generated by the two models and the real plant output are compared to the APRBS input signal for the level of the conical tank 1 is shown in Figure: 8 and Figure: 9 respectively. Analyzing the performance of models, model 2 gives the best open loop and closed loop performance. The best fit criterion is also high in the Model 2 and is shown in Table: 2 . The closed loop N4SID algorithm gives better performance, than the CVA method based on best fit criterion. Based on the pole locations of the models, the open loop methods give biased results. The closed loop N4SID algorithm gives unbiased estimation of system poles which gives capacity to work with the inputs and noise. In the subsequent section, PI controller settings are derived by using combined with the Smith delay compensator and IMC for the control of conical tank system in closed loop. The methodology of the closed loop identification is given in Appendix A.

Table: 2,

Performance comparison of Model 1 and Model 2

\begin{tabular}{cccc}
\hline \hline \multirow{2}{*}{ Structure } & Simulated Output & \multicolumn{2}{c}{ Best fit criterion } \\
\cline { 2 - 4 } & & Model 1 (CVA) & Model 2 (N4SID) \\
\hline \hline \multirow{2}{*}{ Open Loop } & Level of Tank 1 (LT1) - Loop 1 & 72.63 & 78.57 \\
\cline { 2 - 4 } & Level of Tank 1 (LT1) - Loop 2 & 68.58 & 72.32 \\
\hline \hline \multirow{2}{*}{ Closed Loop } & Level of Tank 1 (LT1) - Loop 1 & 71.25 & 82.54 \\
\cline { 2 - 4 } & Level of Tank 1 (LT1) - Loop 2 & 69.84 & 70.93 \\
\hline \hline
\end{tabular}

\section{Smith Predictor based IMC tuned PI Controller Design}

Many process industries have the large dead time. The dead time is significant in the process the PID controller may give lower control performance, this may be re tuned or re designed by using a Smith-predictor for the better servo and regulatory control purpose. 


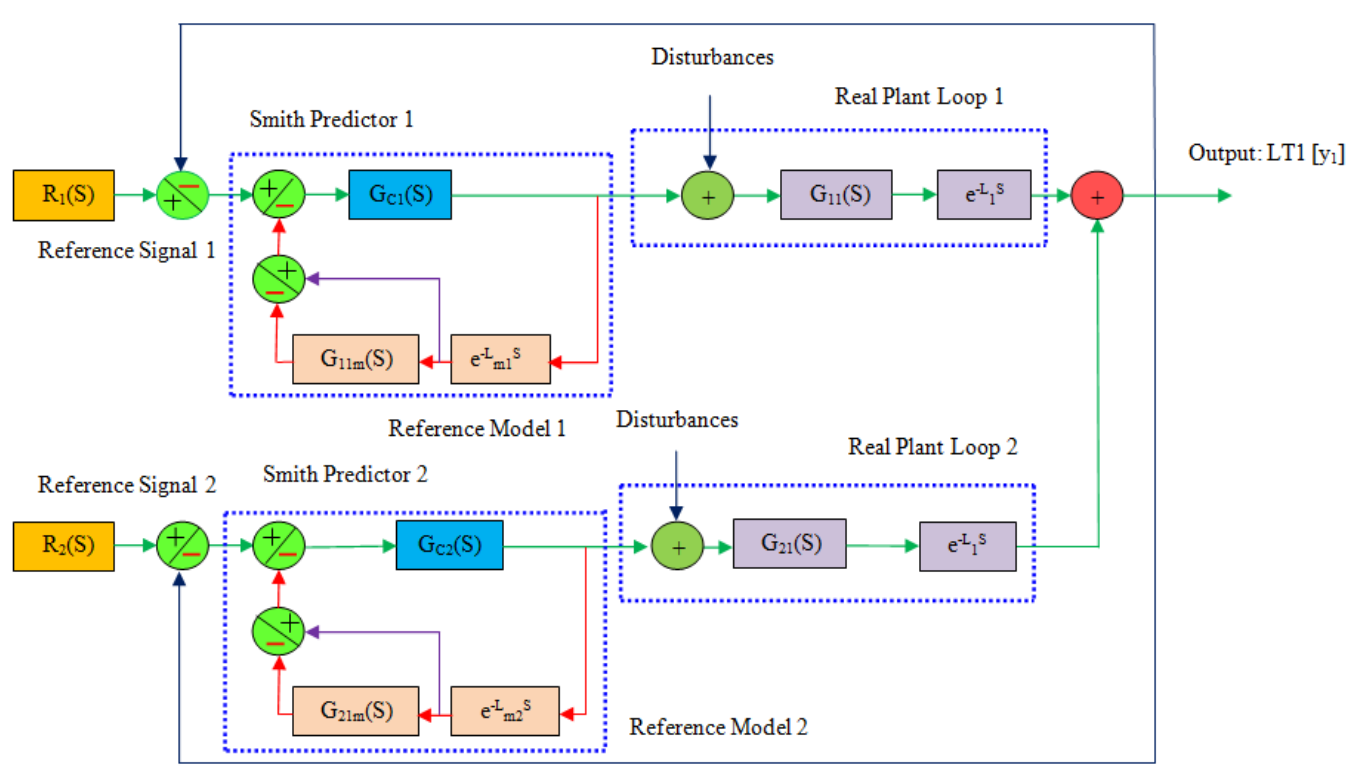

Figure: 10. The structure of Smith Predictor Based IMC-PI control system

The smith-predictor based IMC tuned PI controller with system is shown in Figure: 6, where Gc1(S) \& $\mathrm{Gc}_{\mathrm{c}}(\mathrm{S})$ represent the controller transfer function of Loop 1 and Loop 2 respectively. The mathematical model of the system is represented by a transfer function $G_{p 1}(S)$ \& $G_{p 2}(S)$ is given by $G_{p 1}(S)=G_{11}(S) e^{-l_{1} S} \& G_{p 2}(S)=G_{21}(S) e^{-l_{2} S}$. The reference model is represented by $G_{m l}(S)=G_{11 m}(S) e^{-l_{m l} S} \&$ $G_{m 2}(S)=G_{21 m}(S) e^{-l_{m 2} S}$. The transfer function of the conical tank system with reference to input 1 is given in equation (18)

$\frac{Y_{l}(S)}{R_{l}(S)}=\frac{G_{c l}(S) G_{11}(s) e^{-l_{l} S}}{1+G_{c l}(s)\left(1-e^{-l_{m l} S}\right) G_{11 m}(S)+G_{c l}(S) G_{11}(s) e^{-l_{l} S}}$

The transfer function of the conical tank system with reference to input 2 is given in equation (19) $\frac{Y_{1}(S)}{R_{2}(S)}=\frac{G_{c 2}(S) G_{21}(S) e^{-l_{2} S}}{1+G_{c 2}(S)\left(1-e^{-l_{m 2} S}\right) G_{21 m}(S)+G_{c 2}(S) G_{21}(S) e^{-l_{2} S}}$

If there is no model mismatch, then the process model equal to the real process. The disturbances is negligible, so the closed loop transfer function becomes

$\frac{Y_{1}(S)}{R_{1}(S)}=\frac{G_{c 1}(S) G_{11 m}(S) e^{-l_{m 1} S}}{1+G_{c 1}(S) G_{11 m}(S)}$
$\frac{Y_{1}(S)}{R_{2}(S)}=\frac{G_{c 2}(S) G_{21 m}(S) e^{-l_{m 2} S}}{1+G_{c 2}(S) G_{21 m}(S)}$

The PI controller designed, according to Internal Model Control tuning method for the first order system (Loop 1) is given by

$K_{c}=\frac{1}{K_{p}} \frac{T_{p}}{T_{d}+T_{c}} ; T_{i}=T_{p}$

$\mathrm{K}_{\mathrm{c}}$ is the controller gain, $\mathrm{T}_{\mathrm{i}}$ is the integral time constant and $\mathrm{T}_{\mathrm{c}}$ closed loop constant.

$T_{c}=\max \left\{0.1 T_{p}, 0.8 T_{d}\right\}$

The PI controller designed, according to Internal Model Control tuning method for the second order system (Loop) is given by

$K_{c}=\frac{2 \zeta T_{p}}{K_{p}\left(\lambda+T_{d}\right)} ; \quad T_{i}=2 \zeta T_{p}$ 
$\mathrm{K}_{\mathrm{c}}$ is the controller gain, $\mathrm{T}_{\mathrm{i}}$ is the integral time constant, $\zeta$ is the damping ratio and $\mathrm{T}_{\mathrm{c}}$ closed loop constant. $\lambda=\max \left\{0.25 T_{d}, 0.2 T_{p}\right\}$

The PI settings of the real time plant are tuned according to above mentioned rules and the results are compared conventional Ziegler Nichols tuning method in cynical tank system. The PI settings of the two controllers are shown in Table: 3 .

Table: 3, PI Settings

\begin{tabular}{|c|c|c|}
\hline Tuning method & Controller Transfer & unction matrix \\
\hline \multirow[t]{2}{*}{ ZN method } & $0.063452 S+0.0474$ & $0.1525 S+0.20361]$ \\
\hline & $0.7432 S$ & $0.52376 S$ \\
\hline \multirow[t]{2}{*}{ Smith Predictor Based IMC } & $0.00276 S+0.0765$ & $0.08975 S+0.1032$ \\
\hline & $0.4126 S$ & $0.6126 S$ \\
\hline
\end{tabular}

\section{Control Performance}

The steady state accuracy of the conical tank system is measured by integral square error criterion. The integrated absolute error (IAE) is given by

$$
J_{I S E}=\sum_{k=1}^{N}\left(y(k)-y_{S S}\right)^{2} T_{0}
$$

Where yss is steady state response of the system.

The maximum percent overshoot $(\mathrm{PO})$ of the system is given by

$$
J_{P O}=\frac{\max _{1 \leq k \leq N}\left(y(k)-y_{S S}\right)}{y_{S S}} .100 \%
$$

The performance of the controller is associated with the motion of the actuator. The total variation (TV) is computed as the sum of all manipulated variable movements, which should be minimized.

TV is a measure of control signal smoothness [16] and is given by

$$
T V=\sum_{i=1}^{N}\left|u_{i+1}-u_{i}\right|
$$

\section{Results and Discussion}

The level in the conical tank is controlled by using the two controller settings. The Smith predictor-based IMC-tuning PI controller implemented to the conical tank system, which the system is considered as a MISO configuration. This method is compared to the ZN-PI control algorithm. The performance of both control algorithms are evaluated in computer simulation using the conical tank system. The deviation of level in the tank is shown is shown in Figure: 11.

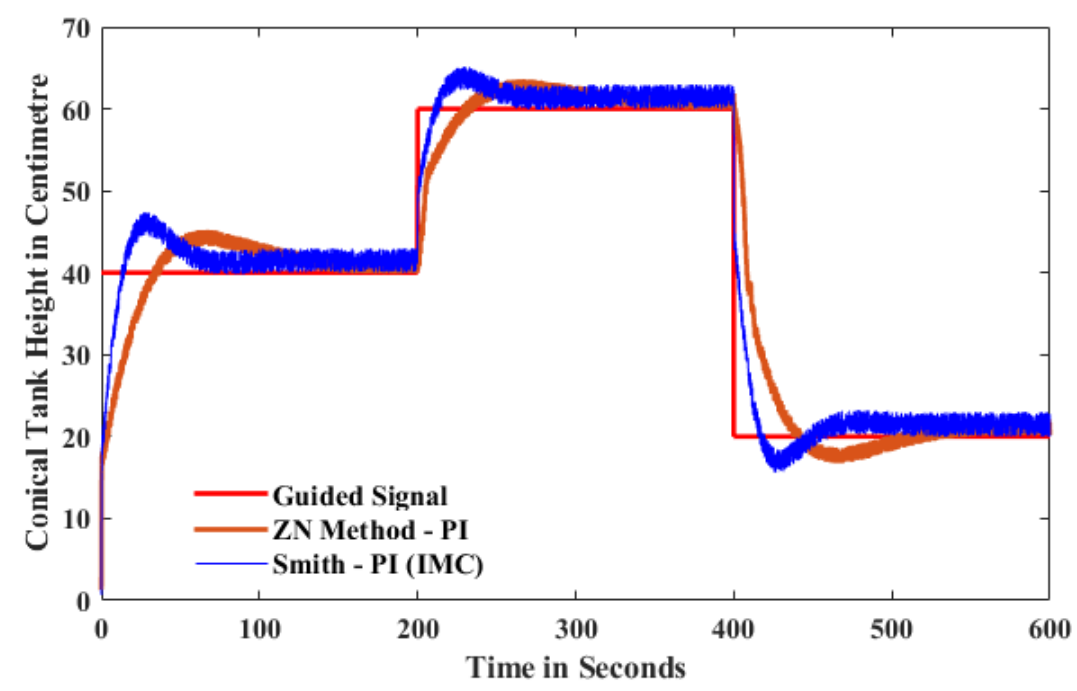

Figure: 11. The variation of level in the tank 1 


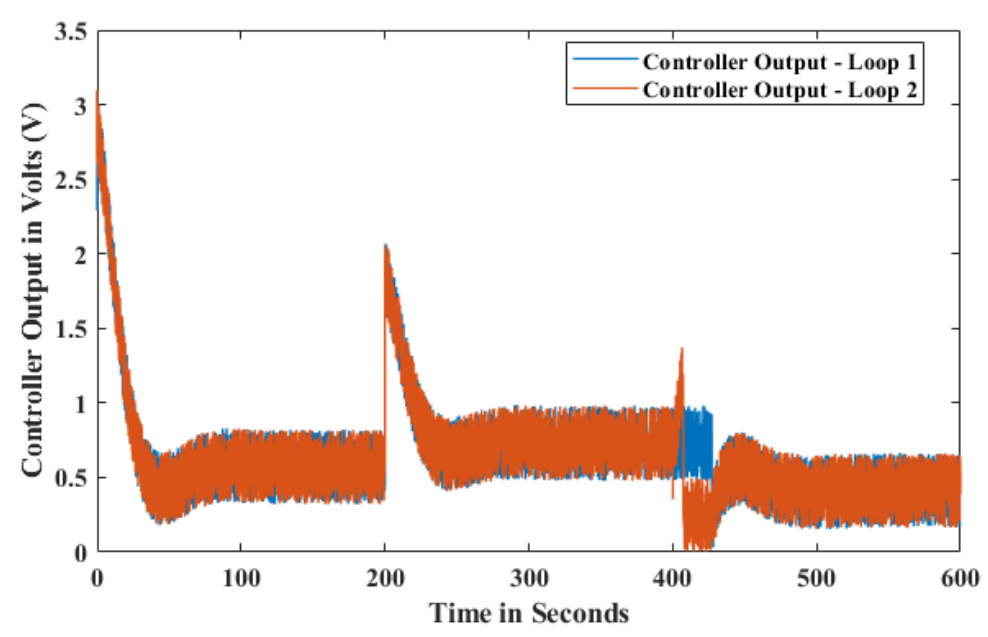

Figure: 12 . The controller output

From the Figure: 11, it is observed that the very little oscillations and the small overshoot and the process variable which level of the tank is capable to track the given set points at $40 \mathrm{~cm}, 60 \mathrm{~cm}$ and 20 $\mathrm{cm}$. The input flow to the conical tank is controlled by PI controller settings, these settings are calculated by $\mathrm{ZN}$ and Smith-IMC tuning methods. The variation in the controller output or input flow rate is shown in Figure: 12.

Table 3

The PI control performance and control effort

\begin{tabular}{|c|c|c|c|c|c|c|}
\hline \multirow{2}{*}{$\begin{array}{c}\text { Transient \& } \\
\text { Performance } \\
\text { Specifications / Tuning } \\
\text { method }\end{array}$} & \multirow{2}{*}{$\begin{array}{l}\text { PO } \\
(\%)\end{array}$} & \multirow{2}{*}{$\begin{array}{l}\text { R.T } \\
\text { (Sec) }\end{array}$} & \multicolumn{2}{|c|}{ ISE } & \multicolumn{2}{|c|}{ TV } \\
\hline & & & Loop 1 & Loop 2 & Loop 1 & Loop 2 \\
\hline ZN method & $\begin{array}{l}13.04 \\
\end{array}$ & 28.06 & 28.35 & 36.48 & 0.62 & 0.99 \\
\hline Smith Predictor Based IMC & 18.37 & 4.21 & 16.12 & 20.54 & 0.67 & 0.83 \\
\hline
\end{tabular}

The output of the controller is varied in plant operation due to the controlled variable variation; however the control action is smooth response even the present of the controlled variable and shown in Figure: 12. The performance of control and control effort is given in Table: 3.

The peak overshoot and settling time taken for the set point $40 \mathrm{~cm}$ is mentioned in table 3 , however the similar characteristics is obtained for subsequent set points $(60 \mathrm{~cm} \& 20 \mathrm{~cm})$. The settling time mentioned in $5 \%$ error criterion. Smith predictor based IMC tuned PI controller better controller performance over $\mathrm{ZN}$ tuning method over settling time, rise time and integral square error. The peak over shoot is slightly lower in ZN method, but not significant performance compared to the proposed method. Due to the over shoot, the total variation is also lower in one loop compared to proposed method, however the TV is also affected control taken by the proposed method. But subsequent set point changes the over shoot similar in both methods. Overall the proposed method gives the better performance than the $\mathrm{ZN}$ tuning method.

\section{Conclusion \& Future Work}

The closed loop N4SID algorithm proposed to identify the MISO dynamic conical tank system. The identified model is a linear and the real time dynamic plant is non linear, but linear model behaves closely to the non linear plant within some bounds. The proposed better validation results and pole assignment over closed loop SVA and open loop methods. Based on the model, the PI controller tuned using smith predictor based IMC tuning method. The efficiency of the proposed method is showed in the conical tan system, compared with ZN tuning method. Further, the PI parameters may be tuned to obtain a robust control using $\mathrm{H} \infty$ based mixed sensitivity algorithms. The 
contribution of the work is on the MIMO state space identification using closed subspace system identification(N4SID) method was executed in the real time conical tank system, and the developed models validated the smith predictor based IMC tuned PI controller on the plant.

Author Contributions: Ganesh Babu C and Sharmila D proposed the idea of this research work and conceived the tests; Sudhahar S performed the tests and wrote the paper. All authors have read and agreed to the published version of the manuscript.

Funding: "This research received no external funding"

Acknowledgments: In this section you can acknowledge any support given which is not covered by the author contribution or funding sections. This may include administrative and technical support, or donations in kind (e.g., materials used for experiments).

Conflicts of Interest: Declare conflicts of interest or state "The authors declare no conflict of interest."

\section{Appendix A}

Process of MISO Configured Conical tank system Model Identification in Closed Loop

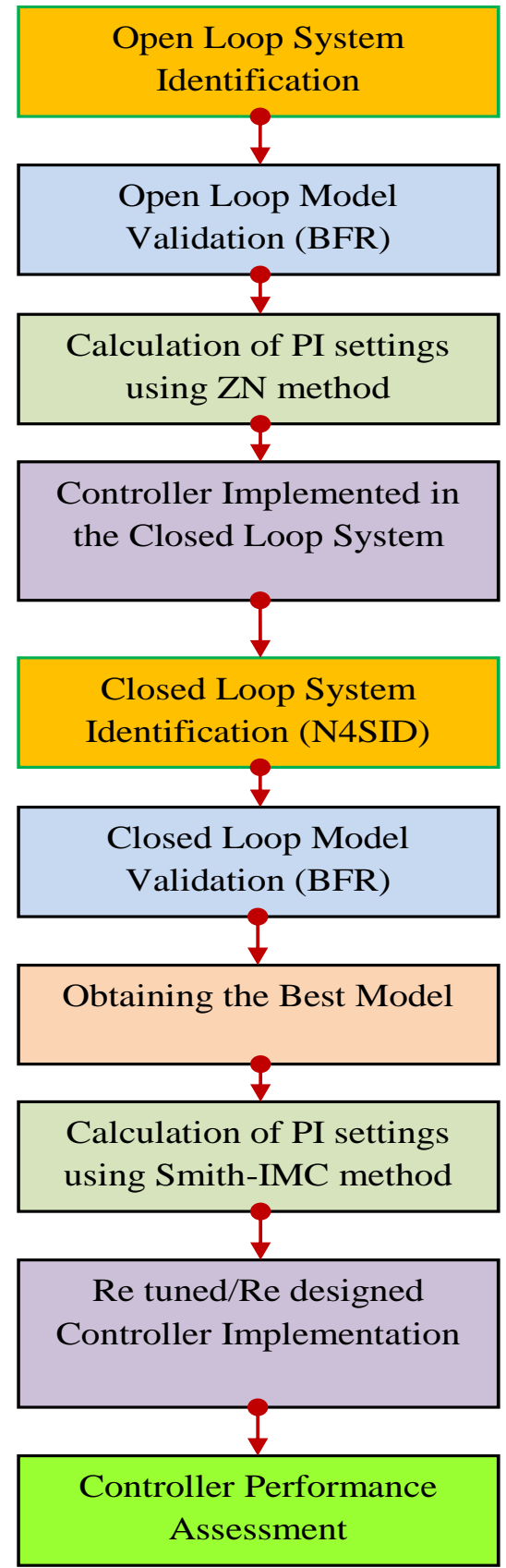




\section{References}

1. Kumar, Abhishankar, Nabil Magbool Jan, and Sridharakumar Narasimhan. "Economically Optimal Input Design Approach for Identification of Constrained Processes." Industrial \& Engineering Chemistry Research 57, no. 20 (2018): 6956-6967.

2. Diversi, Roberto, Roberto Guidorzi, and Umberto Soverini. "Maximum likelihood identification of noisy input-output models." Automatica 43, no. 3 (2007): 464-472.

3. Chen, Juan, Ze-Fang He, and Xin Qi. "A new control method for MIMO first order time delay non-square systems." Journal of Process Control 21, no. 4 (2011): 538-546.

4. Gustavsson, Ivar, Lennart Ljung, and Torsten Söderström. "Identification of processes in closed loop-identifiability and accuracy aspects." Automatica 13, no. 1 (1977): 59-75.

5. Ljung, L. "System identification-theory for the user 2nd edition prentice-hall." Upper Saddle River, NJ (1999).

6. Katayama, Tohru, Hidetoshi Kawauchi, and Giorgio Picci. "Subspace identification of closed loop systems by the orthogonal decomposition method." Automatica 41, no. 5 (2005): 863-872.

7. Kolavennu, S., S. Palanki, and J. C. Cockburn. "Nonlinear control of non square multivariable systems." Chemical Engineering Science 56, no. 6 (2001): 2103-2110.

8. Lamara, Abderrahim, Patrick Lanusse, Guillaume Colin, Alain Charlet, and Yann Chamaillard. "A non-square MIMO fractional robust control for the airpath of a diesel engine." In 2013 European Control Conference (ECC), pp. 3482-3487. IEEE, 2013.

9. Landau, I. D. "Identification in closed loop: a powerful design tool (better design models, simpler controllers)." Control Engineering Practice 9, no. 1 (2001): 51-65.

10. Larimore, Wallace E. "System identification, reduced-order filtering and modeling via canonical variate analysis." In 1983 American Control Conference, pp. 445-451. IEEE, 1983.

11. Lin, Weilu, S. Joe Qin, and Lennart Ljung. "Comparisons of subspace identification methods for systems operating on closed-loop." IFAC Proceedings Volumes 38, no. 1 (2005): 494-499.

12. Li, Kuan, Hao Luo, Chengming Yang, and Shen Yin. "Subspace-aided closed-loop system identification with application to dc motor system." IEEE Transactions on Industrial Electronics 67, no. 3 (2019): 2304-2313.

13. Liu, Jianchang, Nan Chen, Xia Yu, and Shubin Tan. "Modified internal model control for non-square systems based on Smith delay compensator control." Sensors \& Transducers 165, no. 2 (2014): 96.

14. Gong, Qingsong, Genke Yang, and Changchun Pan. "A study of MEWMA controller for drifted MIMO non-squared process." In Proceedings of the 33rd Chinese Control Conference, pp. 2903-2907. IEEE, 2014.

15. Santhosh Kumar, P. L., I. Thirunavukkarasu, Kumar Selva, and Vinayambika S. Bhat. "Smith predictor based pi controller design for a batch distillation column." International Journal of Pure and Applied Mathematics 118, no. 22 (2018): 1109-1115.

16. Skogestad, Sigurd. "Simple analytic rules for model reduction and PID controller tuning." Journal of process control 13, no. 4 (2003): 291-309.

17. Verhaegen, Michel. "Identification of the deterministic part of MIMO state space models given in innovations form from input-output data." Automatica 30, no. 1 (1994): 61-74.

18. Viberg, Mats. "Subspace-based methods for the identification of linear time-invariant systems." Automatica 31, no. 12 (1995): 1835-1851.

19. Wang, Qing-Guo, Xin Guo, and Yong Zhang. "Direct identification of continuous time delay systems from step responses." Journal of Process Control 11, no. 5 (2001): 531-542.

20. Wang, L., and W. R. Cluett. "System identification based on closed-loop step response data." IEE Proceedings-Control Theory and Applications 141, no. 2 (1994): 107-110.

21. Young, Peter, Hugues Garnier, and Marion Gilson. "An optimal instrumental variable approach for identifying hybrid continuous-time Box-Jenkins models." IFAC Proceedings Volumes 39, no. 1 (2006): 225-230. 